\title{
The Reverse-Engineering-based Design of Three-dimensional CAD Parameterized Organic Morphology Mold
}

\author{
Wu Ye \\ School of Architecture, \\ Tianjin University, \\ Tianjin, 300072, China \\ E-mail: yhyewu@yahoo.com.cn
}

\author{
Ruoyu Liang \\ Department of Industrial Design, \\ Tianjin University \\ Tianjin, 300072, China \\ E-mail: lryasa@yahoo.cn
}

\begin{abstract}
Gives the method of 3D CAD parametric design under the guidance of the theory of reverse engineering. Describes the application of the method development process of complex-shaped surface mold. Analysis of the mold characteristics, coordinate measuring equipment accurate and efficient access to the basic outline of the data, the integration of CAD software to design mold forms, re-use processing module directly form processing code, complete mold processing and validation.

Keywords-Reverse Engineering; Complex Morphology;
\end{abstract} Parameterized

\section{INTRODUCTION}

Parametric 3D CAD software reverse engineering product design of complex-shaped surface mold development, is also one of the best ways to improve the efficiency of mold design. The so-called parametric design is the use of the parameters on the size of the product development constraints, in order to achieve the parameters of the graphics driver. Product parametric design can be reflected in the following three aspects: 1.The parameterization using CAD software platform is about to begin design products geometric constraint and engineering constraints; 2.according to the existing parameters related drawing program generated graphics; 3 . to correct reverse generated 3D CAD models based on parameters related constraints. Traditional parametric design is based on the forward design ideas for development work, but when the design stereotypes need to experimentally test the parts models, usually using the method of reverse design.

Currently, parametric design method based on the theory of reverse engineering in the field of mold design has been widely used[1]. Solidworks is a large CAD software, it is able to provide users with a variety of parametric design module designed to meet different needs[2]. This article mainly discusses the software as a working platform.

\section{THE IMPLEMENTATION PROCESS OF PARAMETERIZATION BASED ON REVERSE ENGINEERING 3D CAD MOLD DESIGN}

The overall development process can be divided into three parts: First, the use of coordinate measuring instrument mold prototype lattice-scan, the scan results after their initial treatment, the use of reverse engineering software; then, after preliminary treatment scan results point file to import 3D CAD software, its modeling capabilities will sweep the surface to obtain gradually into the surface, until the part model is generated; Finally, the use of 3D CAD software post-processing module to generate the processing code, the mold processing. As shown in Figure 1.

\section{THE METHOD OF REVERSE ENGINEERING THEORY TO OBTAIN A MOLD PROTOTYPE LATTICE}

A. The analysis of prototype

In this paper, parametric design method of reverse engineering CAD the palm model analysis, then create a digital model, final processing of the digital model mold, the mold will be used in the production of a new type of insulating gloves. Information provided by the upstream firm, the research group clay molds making palms model, the model prototype is selected from the group consisting of volunteers hand-shaped in the 80th percentile of height, weight, and more representative. As shown in Figure 2.

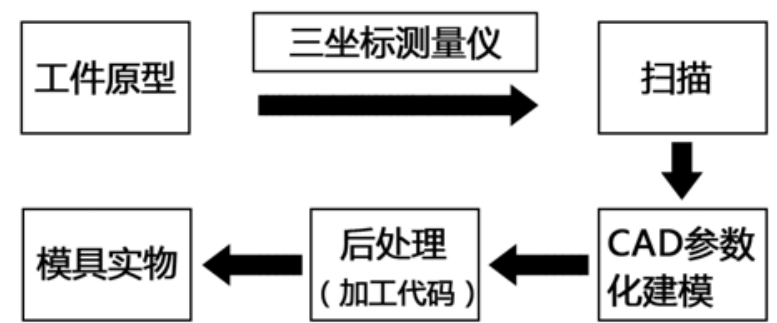

Figure 1. Reverse engineering CAD parametric design flowchart

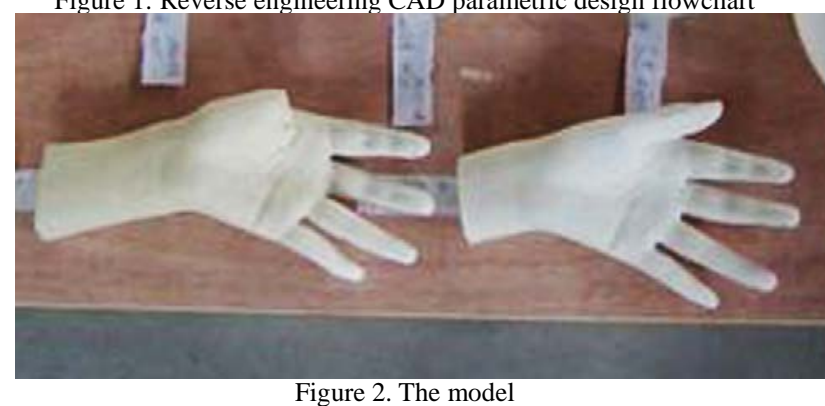

After analysis, the sample form of the model is more complex, and therefore the origin of the coordinate system of the parts can be set at the edge of the sample at the mating surface of the open line scan slice scan to get the coordinates of the model lattice, then use the 3D CAD software reconstruct the final three digits of the sample model.

B. Coordinate measuring instrument collected data 
Under existing conditions, the research group use the TELES YXB-CMM-575-type coordinate measuring instrument scan sample data. The specific procedure is as follows:

Step1. Clamping entities. Check scanning probe, and then take advantage of the multi-purpose fixture clamping model sample work surface coordinate measuring instrument. Model making material is gypsum, soft texture, it is in the clamping process should prevent the model from damage.

Step2. Scanning coordinate system. Established coordinate system to take into account the relationship between the direction of the coordinate system established by the use of CAD software in the direction of the coordinate system with the coordinate measuring instrument. The same direction to ensure that the graphics and graphics export the data displayed in the CAD software. The research group used reverse engineering processing software Geomagic Studio, in its default coordinate system can be adjusted to ensure consistency with Solidworks coordinate system.

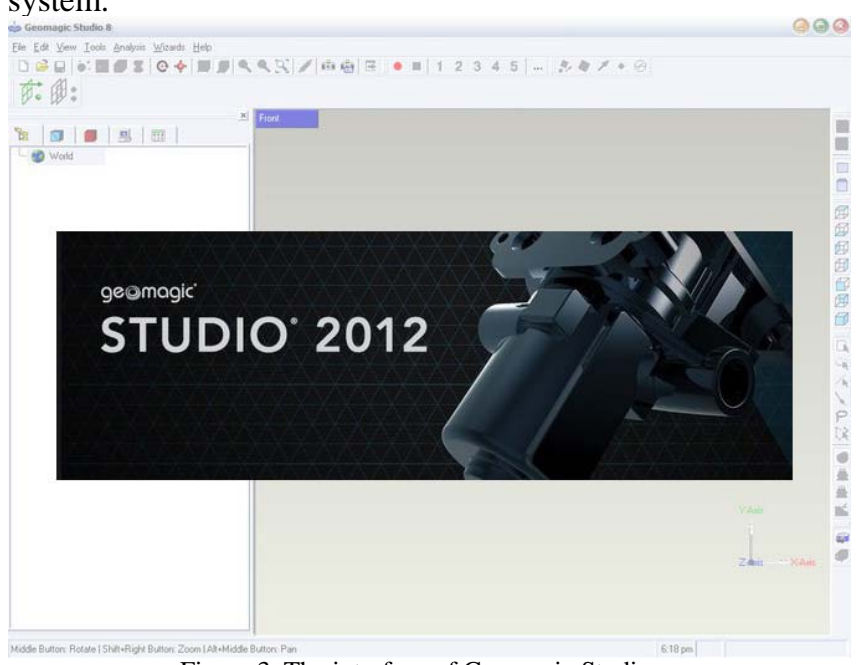

Step3. Choose the scan mode. Coordinate measuring instrument used scanning open line scan, line scan closure, patch scanning cross-section scanning, boundary scan. According to the above analysis, this model sample using the opening line scan with the scan facet practices.

Step4. Data processing. Set of scanning parameters, and then start scanning. Geomagic Studio measurement chamber scan after initial processing to obtain the coordinates of the lattice data.

\section{3D CAD SOFTWARE CONSTRUCTION MOLD MODEL}

Geomagic Studio software processing after the results of the scan are stored as IGES format, and then imported into Solidworks software[3], surface modeling capabilities of the software to build the mold model. The steps are as follows:

Step1. Establish spline curve. Constrained to obtain the parameters of the scan as a whole, using the dot to create a contour drawing guide lines.
Step2. Surfaces. The use of step draw contour lines and guide lines by "lofting" way to generate surfaces. As shown in Figure 4.
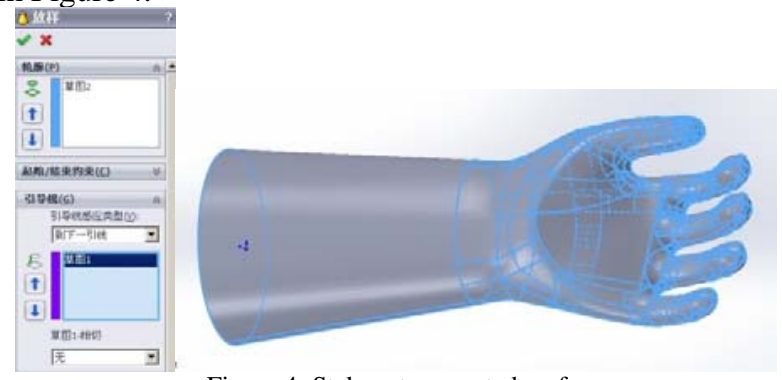

Figure 4. Stakeout generated surface

Step3. Curvature analysis model. According to the error standard, according to the curvature defined parameters, use the "curvature" and "zebra stripes" to test the model. As shown in Figure 5.

After inspection, the palm edge with the wrist bonding part of the curvature of $0.00448567,0.00467864$ finger engaging portion of curvature $0.017067,0.016782$ comply with G2 continuity to meet the design requirements.

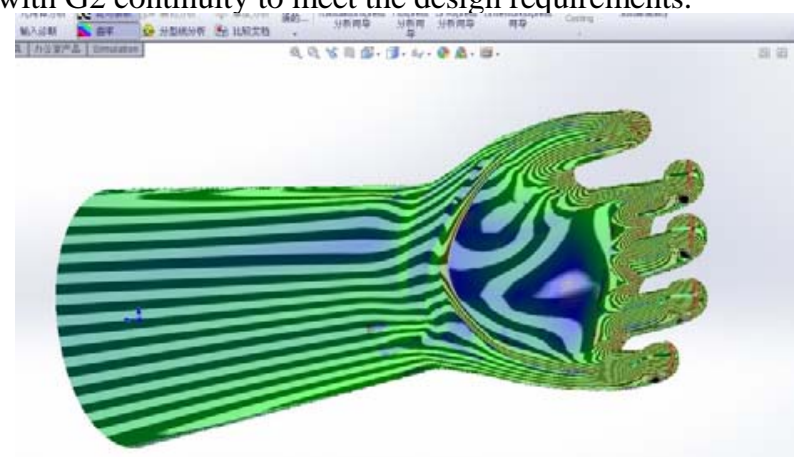

Figure 5. Curvature Analysis

After several adjustments, follow the principles of industrial design, based on the functional requirements of ergonomics, materials, production processes, conditions, and finalize the program.

\section{DESIGN VERIFICATION}

In order to ensure the accuracy of the model, the research group Solidworks post-processing module SolidCAM generates NC code for CNC machining[4]. Processing raw materials for composite nylon material. After rough milling and fine milling axis CNC machining center, access to the products mold sample, the test results of the samples meet the research group quality requirements.

\section{THE ANALYSIS OF ERRORS}

Expanded based on the theory of reverse engineering product design tend to be some errors in the collection of the sample characteristics, these errors are mainly due to the precision coordinate measuring instrument as well as the mode of operation of the laboratory personnel misconduct[5].

Samples on a coordinate measuring instrument scan, scan mode selection, scan step away from the determination of the size, the results of the reverse has a certain impact, 
which determines the size of the scanning step reverse greatest impact, scanning step is too large relative to the error will increase[6], whereas the error will be reduced, but when the scanning step is too small, the scan efficiency will be low, generates data files that will be relatively large[7], and is not conducive to subsequent processing.

For obvious features of the surface parts using coordinate measuring machine, using the parts feature scanning method can simplify the scanning process, reducing the amount of scanned data, to improve the processing speed, and speed up the reverse design process, the method for distinguishing characteristics of the surface parts the reverse design has a certain significance.

\section{SUMMARY}

Parametric CAD design methodology under the guidance of the theory of reverse engineering, it is obvious that the method has great advantages for the construction of complex shaped surfaces part model. Now the new insulated gloves based on the mold have been bring into the market, and get the Chinese State Intellectual Property Office patent, Patent No.: 201130069674.4. The method is quick and reliable, does not require a large number of Surveying and Mapping, programming, and lower for experiment processing equipment requirements, has a high practical value.

\section{REFERENCES}

[1]. Xin Zhang ; Jianwu Zhang, “A cost Estimation Method Based on the Secondary Development of Solidworks”, Journal of Advanced Manufacturing Systems, v 10, n 1, p 85-92, June 2011.

[2]. Dan Su; Jun Shen; Hongmei Zhao, "Parameterized design for the Furniture product based on Solidworks", Advanced Materials Research, v 505, p 402-406, 2012, Manufacturing Engineering and Process.

[3]. Xuemei Li ; Wangbiao Qiu; Shengnan Lv, "Research on quickly data sharing between Geomagic and SolidWorks”, 2010 International Conference on Mechanic Automation and Control Engineering, MACE2010, p 6283-6286, IEEE Press,2010

[4]. Xinglong Liao; Xu Ma; Yanjun Zuo, "Design of rotary root stubble digging machine based on solidworks", IFIP Advances in Information and Communication Technology, v 344 AICT, n PART 1, p 532-538, 2011

[5]. Curtis, S.K.; Harston S.P.; Mattson, C.A., "The fundamentals of barriers to reverse engineering and their implementation into mechanical components”, Research in Engineering Design, v 22, n 4, p 245-61, Oct. 2011

[6]. Romero-Carrillo, P.; Lopez-Alba, E.; Dorado, R.; Diaz-Garrido, F.A., "DIC-CAM recipe for reverse engineering", AIP Conference Proceedings, v 1431, p 30-6, 2012

[7]. Xingwei Liu; Guo-fu Yin; Yang Luo, "Research on reverse engineering system model based on partial surface reconstructing”, Computer Integrated Manufacturing Systems, v 7, n 4, p 12-16, April 2001 